\title{
Investigation of the Effects of Autophagy Signaling on the Transcription of Yeast Retrotransposon Ty2-917
}

\author{
Sezai Turkel ${ }^{1}$ (D), Ceyda Colakoglu² ${ }^{\mathbb{D}}$, Tugce Karaduman ${ }^{1}$ (iD \\ 'Bursa Uludag University, Faculty of Arts and Sciences, Department of Molecular Biology and Genetics, Bursa, Turkey \\ ${ }^{2}$ Bursa Uludag University, Faculty of Medicine, Department of Medical Biology Bursa, Turkey
}

ORCID IDs of the authors: S.T. 0000-0001-7128-6948; C.C. 0000-0002-7471-5071;T.K. 0000-0003-0479-0559

Please cite this article as: Turkel S, Colakoglu C, Karaduman T. Investigation of the Effects of Autophagy Signaling on the Transcription of Yeast Retrotransposon Ty2-917. Eur J Biol 2021; 80(2): 107-118. DOI: 10.26650/EurJBiol.2021.1011143

\begin{abstract}
Objective: Ty2-917 is a low copy retrotransposon found in the Saccharomyces cerevisiae genome. It has structural similarities to metazoan retroviruses in terms of genome organization and propagation mechanisms in the host cells. The objective of this study is to analyze the effects of autophagy signaling on the transcriptional regulation of Ty2 in yeast cells.

Materials and Methods: Ty2-LacZ gene fusions on the YEp vectors have been used as reporter genes to analyze the effects of amino acid starvation, nitrogen source, and autophagy signals on the transcription of Ty2. These reporter gene fusions have been transformed into the wild type and also isogenic mutant yeast strains that are defective for one of the regulatory factors involved in nutrient sensing and signaling. To activate autophagy signaling, yeast transformants were treated with caffeine or 3-amino 1-2-3 triazole. Transcription levels of Ty2-LacZ gene fusions in treated and untreated yeast cells were analyzed by $\beta$-galactosidase assays.

Results: Results of this study show that transcription of Ty2 decreases up to eightfold in response to amino acid starvation. Caffeine treatment of the yeast cells also represses Ty2 transcription, independent of the TOR1 pathway. In addition, our results suggest that Ty2 transcription is also regulated in a nitrogen source-dependent manner through the GATA factors.

Conclusions: Our results suggest that activation of autophagy signal results in significant level repression of Ty2 transcription. We have found that the GATA class of transcription factors is involved in the regulation of Ty2 transcription in response to autophagy signaling.
\end{abstract}

Keywords: Autophagy, Transcription, Yeast, Retrotransposons, GATA factors

\section{INTRODUCTION}

The Saccharomyces cerevisiae genome has five different types of mobile genetic elements known as Ty (Transposon Yeast) (1). Ty elements propagate via the RNA intermediate in the yeast genome. They form virus-like particles (Ty-VLP) in the yeast cytoplasm (2). Their genome organization and intracellular propagation mechanisms show a large degree of similarities to metazoan retroviruses (3). They are called retrotransposon due to these similarities. The taxonomic classifications of yeast retrotransposons in the world of viruses have also been assigned. Ty1 and Ty2 are classified in the Pseudovirus genus of the Pseudoviridae family in the Retrovirales order, while Ty3 is included in the Metavirus genus of the Metaviridae family (4).

Ty2-917 (hereafter referred to as Ty2) is one of the retrotransposons found in the yeast genome. It has been discovered as an insertional element within the HIS4 gene of S. cerevisiae (5). Later it was found that it is present as five to ten copies in most of the $S$. cerevisiae laboratory strains (1). Its genome size is $5.9 \mathrm{Kbp}$ and it contains $0.33 \mathrm{Kbp}$ long terminal repeats (LTR) at its $5^{\prime}$ and $3^{\prime}$ ends. These LTRs are called delta elements (Figure 1). It has been shown that the intracellular copy numbers 
of Ty retrotransposons are regulated at post-translational levels and do not change drastically in standard laboratory strains of S. cerevisiae $(6,7)$.

Ty genomes are highly expressed. Single types of polyadenylated mRNA are transcribed from the Ty genomes by RNA polymerase II. It is known that Ty mRNAs constitute up to $10 \%$ of total mRNA in the yeast cytoplasm (8). Ty mRNAs have two open reading frames, called TYA and TYB. These coding regions functionally show homology to retroviral Gag and Pol polypeptides, respectively $(9,10)$. TYA peptide forms the capsid part of the Ty-VLP after post-translational processing. TYB polypeptide is proteolytically processed and yields the reverse transcriptase, protease, and integrase enzymes required for the replication cycle of Ty elements. TYB is translated as a fusion protein to TYA by programmed ribosomal frameshift (PRF) (Figure 1) $(9,10)$.

Promoter regions of Ty2 retrotransposons have multiple overlapping transcription factor binding sites. These regulatory elements are located both upstream and downstream of the transcription initiation site within the $5^{\prime}$ LTR region (11-13). Ty elements do not encode any transcription factors and their transcription is regulated by host-encoded transcription factors. Transcription factors that are involved in the regulation of Ty2 transcription have been identified previously by biochemical and genetic techniques. These factors are; Gcr1, Sgc1, Tec1, Rap1, and chromatin modification complexes such as histone acetyl transferases (14-17). It has previously been reported that

a)

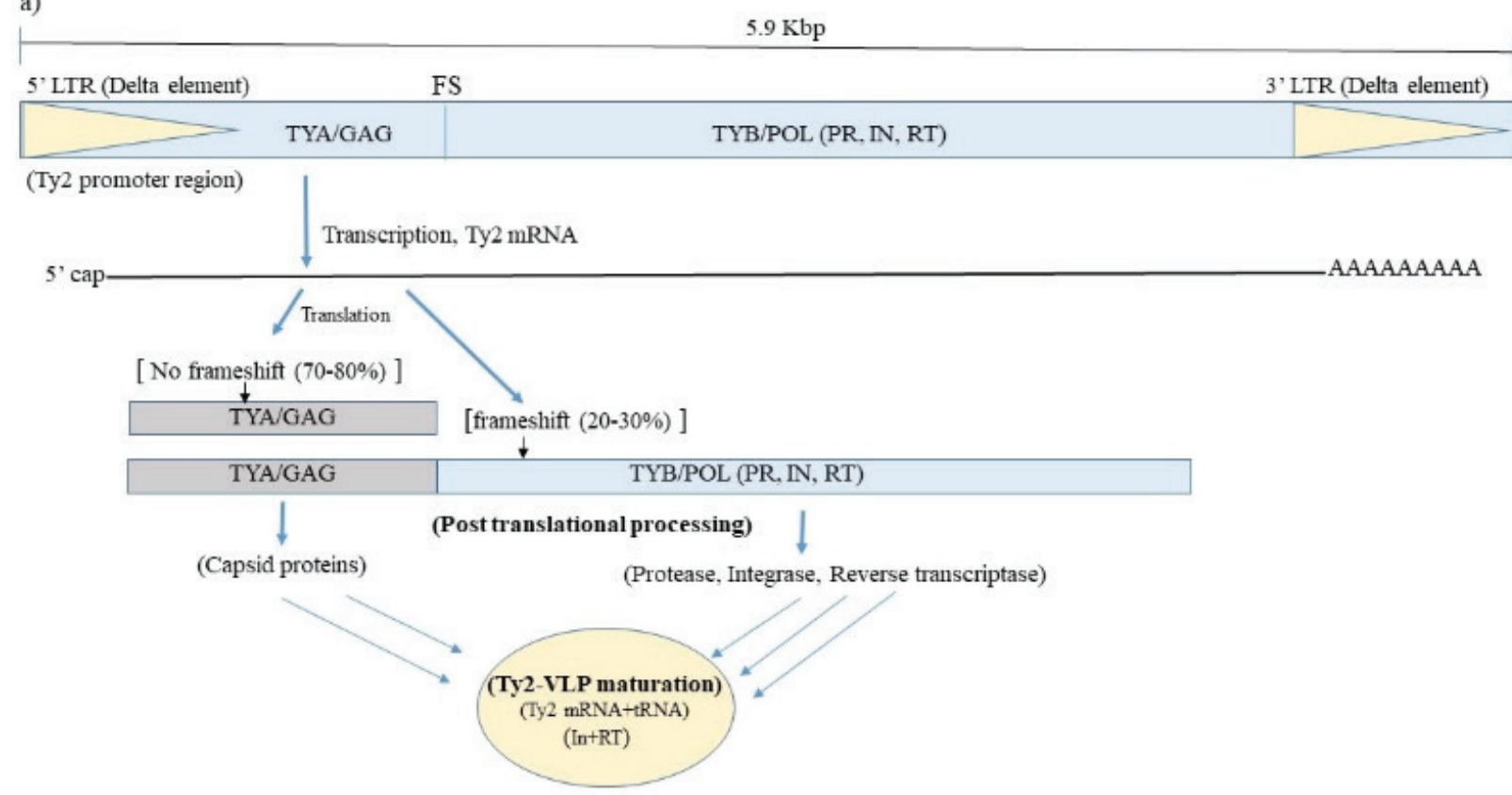

b)

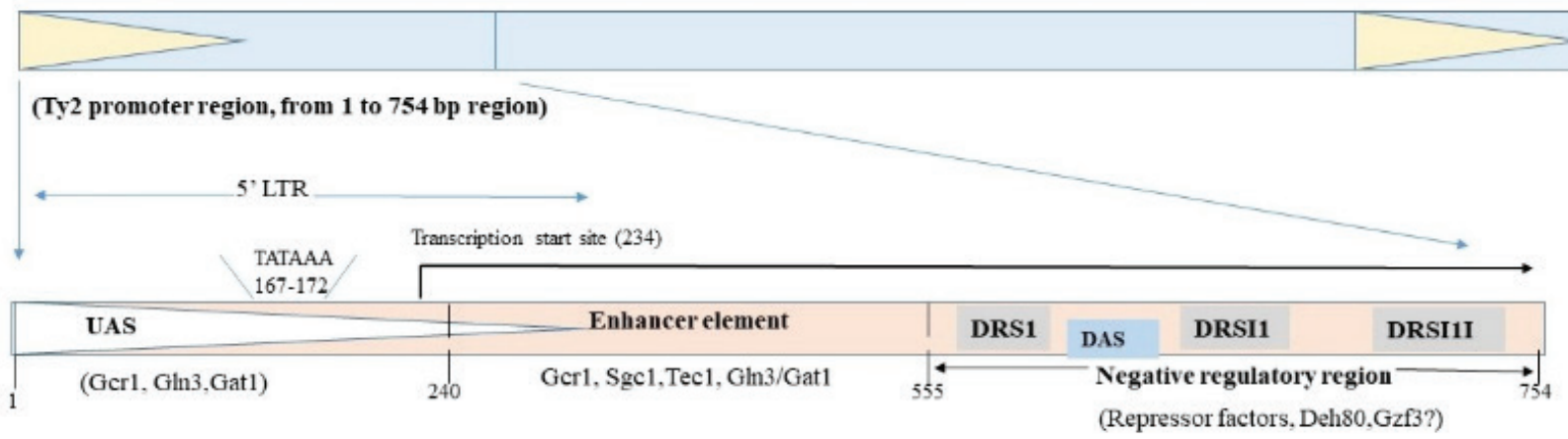

Figure 1. Genome organization and promoter structure of retrotransposon Ty2. a-) Overall genetic structure of Ty2. b-) Organization of functional regulatory region of Ty2 promoter. Abbreviations: FS: Frameshift Site. DRS: Downstream Repression Sites. DAS: Downstream Activation Sites. UAS: Upstream Activation Sequence. 
transcription of Ty elements is regulated in response to metabolic signals. Ty2 transcription is controlled by the transcription factor Gcr1 in response to glucose signaling (17). It seems that Gcr1 is the major transcription factor that activates Ty2 transcription since deletion of the GCR1 gene completely abolishes Ty2 expression (17). Recent evidence indicates that Gcr1 is present as two isoforms. These are $\mathrm{Gcr} 1^{\mathrm{U}}$ and $\mathrm{Gcr} 1^{\mathrm{s}}$. These isoforms show differential stability in the cytoplasm, depending on the growth stage of the yeast cells $(18,19)$.

It is known that activation of selective autophagy targets Ty1VLP in yeast and down-regulates Ty1 mediated insertional mutagenesis (20). Moreover, we have recently reported that transcription in Ty1 and Ty2 is differentially regulated by apoptosis-inducing growth conditions (21).

Autophagy has been defined as the lytic cellular process for macromolecule recycling in adverse conditions. It is conserved in all eukaryotes and essential for cellular homeostasis. Molecular components of the autophagy process have been elucidated by Yoshinori Ohsumi using S. cerevisiae as the model eukaryotic system $(22,23)$. Autophagy-related (ATG) genes that involve in the initiation and execution of the autophagy process have been identified and functionally analyzed in yeast (23). The target of rapamycin (TOR), AMP-activated protein kinase Snf1, also functions in the initial stages of autophagy signaling. Under nutrient replete growth conditions, TOR1 kinase is active and represses the expression of ATG genes (24-28). When TOR1 kinase is inhibited by rapamycin, caffeine treatment, or nutrient-limited growth conditions, the autophagy pathway resumes forming autophagosomes (24-28). Different sets of transcription factors are involved in the regulation of ATG genes. Some of these transcription factors are Ume6, Pho23, Rim15, Gcn4, Gln3, Gat1, and Yap1 (25). Moreover, it is known that Gcn4p, GIn3p, Gat1p, and Yap1 $p$ are also involved in the transcriptional activation of numerous yeast genes, including Ty elements $(29,30)$.

Autophagy can be activated by intracellular and extracellular factors. Intracellular events that activate autophagy are misfolded protein accumulations and damaged or dysfunctional cell components. Extracellular factors that activate autophagy are different chemicals and environmental cues such as amino acid starvation and nitrogen limitations (31, 32). Autophagy processes can be classified into different sub-groups depending on reaction mechanisms. These are macro-autophagy, micro-autophagy, and chaperone-mediated autophagy. In all three of these autophagy processes, targeted macromolecules are transported into lysosomes (in vacuole for yeast) for degradation. In macro-autophagy, targeted macromolecules are transported to lysosomes via autophagosomes that are double membraned cytoplasmic structures sequentially formed by Atg factors (32). On the other hand, in micro-autophagy, targeted cytoplasmic structures are directly taken up by lysosomes. In chaperone-mediated autophagy (CMA), targeted macromolecules are first recognized and complexed with chaperon proteins, then taken up by lysosomes via lysosome-associated membrane protein 2A (LAMP-2A) (32). In certain cases, the above-mentioned autophagy processes selectively target specific cellular structures via binding to specific receptors located on targeted cytoplasmic organelles. This type of autophagy is known as selective autophagy and requires specific interactions between one of the components of the autophagy process and a specific receptor on the targeted cytoplasmic structures. Mitophagy, pexophagy, reticulophagy, and ribophagy are well-known examples of selective autophagy (32).

Previously, it was found that the activation autophagy process reduces Ty1 transposition by decreasing the cytoplasmic Ty1VLP load in S. cerevisiae (20). It was shown that Ty1-VLP was selectively transported to the vacuole in Cvt vesicles (Cytoplasm to vacuole) by Atg19-dependent manner in Rapamycin treated yeast cells. Atg19 is a receptor protein that specifically carries targeted structures to Cvt vesicles for degradation at vacuole (33). It was concluded that the selective autophagy process is operated to prevent or to decrease insertional mutagenesis caused by Ty elements within the yeast cells. Autophagy is also involved in the cytoplasmic virus particle destruction in human cells $(34,35)$. Hence, especially for latent virus particles, autophagy activation has been of interest for potential therapeutic approaches for the eradication of certain human viruses (34, 35). In this study, we have analyzed the effects of autophagy activation on the transcriptional regulation of retrotransposon Ty2. We have shown that activation of the autophagy process downregulates Ty2 transcription at a significant level. Moreover, it appears that TORC1-regulated transcription factors Gln3/Gat1 is involved in the transcriptional regulation of Ty2.

\section{MATERIALS AND METHODS}

\section{S. cerevisiae Strains and Ty2 Expression Vectors}

The genotypes of S. cerevisiae strains used in this study were given in Table 1. The standard laboratory strain of S. cerevisiae BY4741 and its deletion mutants were purchased from the EUROSCARF yeast collection. They are isogenic to each other except for the indicated mutations (36). S. cerevisiae strains F212 and F113 were used to analyze the effects of amino acid starvation on Ty2 transcription. They are wild type for histidine biosynthesis and commonly used to test the effects of 3-amino triazole-triggered effects of amino acid starvation on yeast genes. S. cerevisiae $\mathrm{F} 212$ and F113 strains are also isogenic pairs other than gcn4 mutations (Table 1). S. cerevisiae strains YST182 and YST184 were used to test the effects of transcription factors Gln3/Gat1 on Ty2 transcription (37). Yeast strains were grown in YPD (1\% Yeast Extract, 2\% Pepton, 2\% Dextrose) medium for routine cultivations in a $30 \mathrm{C}$ incubator shaker (150 rev/min). To confirm the deletions in mutant strains and the presence of KanMX deletion cassette, mutant strains were streaked and grown on the YPD media containing Geneticin $(200 \mu \mathrm{g} / \mathrm{mL})(36)$.

The characteristics of the yeast expression vectors used in this study were summarized in Table 2 . Structures and construction of plasmids pST1, Ty2-555-LacZ, Ty2-754-LacZ, and pST1-EnhLacZ were described previously (12-14). Expression vector pF$\mathrm{N} 8 \mathrm{X}-\mathrm{n}$ is a $2 \mu$-URA3-based plasmid bearing a fusion of the yeast 
Table 1. Saccharomyces cerevisiae laboratory strains used in this research.

\section{EUROSCARF \\ Accession numbers (relevant mutations)}

\begin{tabular}{|c|c|}
\hline $\begin{array}{l}\text { Y00000 (wild type) } \\
\text { (BY4741) }\end{array}$ & MATa, his $3 \Delta 1$; leu2 $\Delta 0$; met $15 \Delta 0$; ura3 $\Delta 0$. \\
\hline Y06864(Ator1) & MATa, his $3 \Delta 1$; leu2 $\Delta 0$; met15 $\Delta 0$; ura3 $\Delta 0$, YJR066w::kanMX4 \\
\hline $\mathrm{Y} 14311(\Delta s n f 1)$ & MATa, his $3 \Delta 1$; leu2 $\Delta 0$; met15 $\Delta 0$; ura3 $\Delta 0$, YDR477w::kanMX4 \\
\hline Y07155( $\Delta$ tec 1$)$ & MATa, his $3 \Delta 1$; leu2 $\Delta 0$; met15 $\Delta 0$; ura3 $\Delta 0$, YBR083w::kanMX4 \\
\hline$Y 01641(\Delta s g c 1)$ & MATa, his $3 \Delta 1$; leu2 $\Delta 0$; met15 $\Delta 0$; ura $3 \Delta 0$, YOR344c::kanMX4 \\
\hline $\mathrm{F} 212^{*}(\Delta g c n 4)$ & $\begin{array}{l}\text { MATa, ura3-52, ino1 gen4- } \Delta 1 \text { 'Kpn1-Mlu1' (wild type for histidine } \\
\text { biosynthesis) }\end{array}$ \\
\hline F113* (Wild type for GCN4) & $\begin{array}{l}\text { MATa, ura3-52, ino1 (wild type for histidine biosynthesis) } \\
\text { (F212 and F113 strains are also isogenic pairs other than gen4 mutations) }\end{array}$ \\
\hline YST182** (Wild type for GATA factors) & MATa, ade $2 \Delta::$ hisG, his $3 \Delta 200$, leu $2 \Delta 0$, lys $2 \Delta 0$, met $15 \Delta 0, \operatorname{trp} 1 \Delta 63$, ura $3 \Delta 0$. \\
\hline YST184 (03167b)( $\Delta g \ln 3 / \Delta g a t 1$ double mutant) & $\begin{array}{l}\text { MATa, ade } 2 \Delta:: \text { hisG, his } 3 \Delta 200 \text {, leu2 } \Delta 0 \text {, lys } 2 \Delta 0 \text {, met } 15 \Delta 0 \text {, } \operatorname{trp} 1 \Delta 63 \text {, ura3 } \Delta 0 \text {, } \\
\text { gln3::KanMX4, gat1:: KanMX4 }\end{array}$ \\
\hline
\end{tabular}

*S. cerevisiae F212 and F113 strains are from Prof. Dr. Alan Hinnebusch's yeast collection (NIH, Bethesda, Maryland. USA)

${ }^{*}$ S. cerevisiae YST182 and YST184 strains are from Prof. Dr. Evelyne Dubois's yeast collection (Université Libre de Bruxelles, Belgium.)

Table 2. Lists of plasmids used in this study.

\begin{tabular}{ll}
\hline Plasmids/Gene fusions & Relevant characteristics \\
\hline pST1 & $\begin{array}{l}\text { His4-LacZ fusions to codon } 32 \text { of HIS4. The HIS4 transcriptional activators are replaced by } \\
\text { a polylinker containing Xho1, Not1, Kpn1, BamH1 sites. Does not contains any activator } \\
\text { binding sites. (YEp, } 2 \mu-U R A 3) .\end{array}$ \\
\hline Ty2-555-LacZ & $\begin{array}{l}\text { Ty2-917-LacZ fusion at position } 555 \text { of Ty2-917, includes entire enhancer element of } \\
\text { Ty2-917. (YEp, } 2 \mu-U R A 3) .\end{array}$ \\
\hline Ty2-754-LacZ & $\begin{array}{l}\text { Ty2-917-LacZ fusion at position 754 of Ty2-917, includes entire enhancer element and } \\
\text { downstream negative regulatory region of Ty2-917. (YEp, 2 } \mu-U R A 3) .\end{array}$ \\
\hline pST1-Enh-LacZ & $\begin{array}{l}\text { His4-LacZ fusions to codon 32 of HIS4. The HIS4 transcriptional activators are replaced by a } \\
\text { polylinker into which the Ty2-917 enhancer (240-559) was inserted. (YEp, 2 } \mu-U R A 3) .\end{array}$ \\
\hline $\begin{array}{l}\text { His4-LacZ } \\
\text { (pFN8X-n) }\end{array}$ & His4-LacZ fusion, includes entire promoter region of HIS4 gene (YEp2 $\mu-U R A 3)$. \\
\hline
\end{tabular}

wild type HIS4 gene to the E.coli lacZ gene (38). Expression of the His4-LacZ fusion on this plasmid is induced in response to amino acid starvation by GCN4 (39). All plasmids used in this research are the yeast episomal plasmid (YEp)-based expression vectors. YEp plasmids are the shuttle vector that is widely used for the cloning, transformation, and expression of a variety of genes in S. cerevisiae (40). It has been previously shown that the copy numbers of these YEp plasmids do not change drastically in the yeast cells grown in the selective growth media $(11,12)$.

\section{Yeast Transformation}

S. cerevisiae strains were cultivated in $25 \mathrm{~mL}$ YPD medium at standard growth conditions (30 C in incubator shaker, $150 \mathrm{rev} /$ min) to the logarithmic stage for transformation. The lithium acetate-polyethylene glycol method was used for the transformation of Ty2-LacZ expression vectors to the competent S. cerevisiae cells as described (41). Transformants of S. cerevisiae strains with BY4741 genetic background were plated onto synthetic complete media (100 $\mu \mathrm{L}$ ) without uracil (Sc-Ura, 2\% dextrose) and transformation plates were incubated at $30 \mathrm{C}$ incubator for 3-4 days for the growth of transformant yeast colonies. Transformants of S. cerevisiae strains F212 and F113 were plated and maintained on the minimal yeast nitrogen base (YNB) medium supplemented with $2 \%$ glucose. Transformants of S. cerevisiae strains YST182 and YST184 were also plated on YNB medium 
supplemented with $2 \%$ glucose and auxotrophic requirements (20 mg/L histidine, 30mg/L leucine, 30mg/L lysine, $20 \mathrm{mg} / \mathrm{L}$ methionine, and $20 \mathrm{mg} / \mathrm{L}$ tryptophan) as described (37). The yeast transformants were stored at $4 \mathrm{C}$ during the experiments. They are used for inoculation of liquid cultures that are used for autophagy experiments as described below.

\section{Activation of Autophagy Signaling}

For activation of autophagy conditions in BY4741-derived S. cerevisiae strains, yeast transformants first were grown to saturation in $10 \mathrm{~mL}$ of liquid Sc-Ura medium supplemented with $2 \%$ glucose and then diluted to $\mathrm{OD}_{600}: 0.1$ in $10 \mathrm{~mL}$ of fresh Sc-Ura medium supplemented with $2 \%$ glucose. Once the cell density reached the mid-logarithmic stage $\left(\mathrm{OD}_{600}: 1.0\right)$ in these cultures, yeast cultures were divided into two aliquots, $5 \mathrm{~mL}$ each. Filtersterilized caffeine was added to $8 \mathrm{mM}$ final concentration to one aliquot (42). Caffeine-treated and untreated yeast cultures were further incubated for 5 hours at standard growth conditions.

For amino acid starvation experiments, S. cerevisiae strains F212 and F113 transformants first were grown to saturation in $5 \mathrm{~mL}$ liquid YNB media supplemented with $2 \%$ glucose and then diluted to $\mathrm{OD}_{600}: 0.1$ in fresh $10 \mathrm{~mL}$ of YNB liquid media. Once the cell density reached $\mathrm{OD}_{600}: 0.4-0.5$, yeast cultures were divided into two aliquots, $5 \mathrm{~mL}$ each. Filter-sterilized 3-amino 1,2,4 triazole (3-AT) was added to $10 \mathrm{mM}$ final concentration to one portion (29). 3-AT treated and untreated yeast transformants were grown for an additional 10 hours and harvested for $\beta$-galactosidase assays.

In order to analyze the effects of GATA factors on the transcriptional regulation of Ty2, S. cerevisiae YST182 and YST184 transformants were grown in $5 \mathrm{~mL}$ YNB minimal medium supplemented with auxotrophic requirements and different nitrogen sources as described (37). S. cerevisiae strains grown to logarithmic stages at standard growth conditions and then harvested for $\beta$-Galactosidase assays.

At the end of growth periods, yeast cells were harvested by centrifugation and washed twice with $1 \mathrm{~mL}$ of sterile distilled water. After final wash and centrifugation, harvested yeast pellets were resuspended in $200 \mu \mathrm{L}$ of $\beta$-Galactosidase breaking buffer and used for $\beta$-Galactosidase assays as described in the next section. Yeast transformants were grown in duplicates and all experiments were repeated at least twice.

\section{$\boldsymbol{\beta}$-Galactosidase Enzyme Assay}

In order to get yeast cell lysates, $20 \mu \mathrm{L}$ chloroform and $20 \mu \mathrm{L}$ $0.1 \%$ SDS was added to the yeast suspensions and vortexed vigorously for 1 minute to obtain yeast lysates (27). $\beta$-Galactosidase activities of the yeast lysates were determined in triplicates as described previously $(11,43)$. Protein concentrations in the yeast lysates were determined by the Lowry test (44). $\beta$-Galactosidase units are expressed in nano moles of 2-Nitrophenyl $\beta$-D-Galactopyranoside (ONPG) cleaved per minute per mg of protein in the permeabilized yeast cell suspensions (nm ONPG/ minute/ mg protein). Standard deviations for $\beta$-Galactosidase units were below $10-15 \%$ in triplicate assays. Statistical signif- icance ( $p$-values) of the results were calculated using a T-test. The expression levels of gene fusions under normal growth versus autophagy induced conditions in the wild type and mutant yeast transformants were compared using a T-test. Transcription levels were found significantly different $(p<0.05)$.

\section{In-Silico Analysis of the Ty2 and GCR1 Genes Promoter Regions}

Experimentally proven and putative binding sites for Gcr1 and GATA factors were analyzed using YEASTRACT (www.yeastract. com) and Saccharomyces Genome Database (SGD) (www.yeastgenome.org) search tools. The promoter region of Ty2-917 encompasses the first 800 bp region of the Ty2 sequence and contains all of the known regulatory sites (11, 14-17). GCR1 gene has an unusually long, alternatively spliced intron region. The first exon of the GCR1 gene contains only 8 base pairs. The promoter region of the GCR1 gene (Systematic Name: YPL075W) on chromosome XVI has been re-defined as $1 \mathrm{kbp}$ long DNA region upstream of the first ATG codon of first exon (www.yeastgenome.org). Promoter region sequences of the Ty2 and GCR1 gene were downloaded from the SGD database. Potential binding sites for Gcr1 and GATA factors were screened using YEASTRACT transcription factor binding site analysis tools.

\section{RESULTS}

\section{Effect of Autophagy Activation on Ty2-917 Transcription}

We have analyzed the effects of autophagy activation on the transcription of Ty2 retrotransposon and its regulator region, namely the enhancer element. It has previously been reported that autophagy signaling can be generated by caffeine (42). Ty2-754-LacZ gene fusion contains all of the known regulatory regions of Ty2 retrotransposon (Figure 1). Hence we have used this Ty2-754-LacZ gene fusion to test the effects of autophagy activation. Transcription from this Ty 2 gene fusion yielded 185 units of activity in the wild type $S$. cerevisiae grown in a normal growth medium. Activation of autophagy signaling by caffeine treatment resulted in a threefold decrease in the transcription of Ty2 in this yeast strain (Table 3). After showing that the caffeine treatment represses the Ty2 transcription, we wanted to determine the molecular components of the caffeine-dependent repression of Ty2 transcription. Therefore, we have analyzed the effect of caffeine treatment on the mutant strains that lack one of the regulatory factors involve in the TOR signaling or Ty2 regulation. Assuming that the caffeine-triggered autophagy process acts through one of these regulatory factors, we should not see any difference in the transcription of Ty2 in caffeine-treated and untreated mutant yeast cells.

Previous studies have shown that the TOR1 complex (TORC1) is the main factor for the suppression of autophagy in nutrient-replete growth conditions $(32,45)$. Hence, the inactivation of TORC1 initiates the autophagy process in all eukaryotic cells. Snf1 (also known as AMP-activated kinase) involves in the regulation of numerous metabolic events in eukaryotes (46). Transcription of Ty2 decreased in $\Delta$ tor and $\Delta s n f 1$ mutant yeast strains, indicating that functional TORC1 and Snf1 complex in- 
Table 3. Activation of autophagy represses Ty2-754 transcription.

\begin{tabular}{|c|c|c|}
\hline \multirow{2}{*}{ S. cerevisiae strains (relevant mutations) } & \multicolumn{2}{|c|}{$\begin{array}{c}\text { Growth Conditions and } \\
\beta \text {-Galactosidase Activities * }( \pm \text { SD) }\end{array}$} \\
\hline & $\begin{array}{l}\text { Sc-Ura+\%2 Glucose } \\
\text { (Normal growth) }\end{array}$ & $\begin{array}{l}\text { Autophagy induction } \\
\text { (+8 } \mathrm{mM} \text { Caffein) }\end{array}$ \\
\hline Y0000 (Wild type) ${ }^{* *}$ & $185 \pm 15$ & $69 \pm 2$ \\
\hline Y06864 $(\Delta \text { tor } 1)^{* *}$ & $156 \pm 2$ & $86 \pm 2$ \\
\hline $\mathrm{Y} 14311(\Delta s n f 1)^{* *}$ & $116 \pm 1$ & $30 \pm 1$ \\
\hline Y07155 $(\Delta t e c 1)^{* *}$ & $157 \pm 1$ & $54 \pm 4$ \\
\hline Y01641 $(\Delta s g c 1)^{* *}$ & $74 \pm 4$ & $29 \pm 1$ \\
\hline \multicolumn{3}{|c|}{$\begin{array}{l}( \pm \text { SD): Standard deviations. } \\
\text { *Numbers indicate } \beta \text {-Galactosidase Units which are expressed in nano moles of ONPG cleaved per minute per mg of protein. } \\
\text { **Transcription levels were found significantly different }(p<0.05) \text {. }\end{array}$} \\
\hline
\end{tabular}

volved in the transcriptional activation of Ty2 under nutrient-replete conditions (Table 3). Caffeine treatment of these mutant strains resulted in a further decrease in Ty2 transcription. It is noticeable that Ty 2 transcription was repressed nearly fourfold in the $\Delta s n f 1$ strain (Table 3 ). In addition to these protein kinases, we have also tested the effects of transcription factors Tec1 and Sgc1 on the Ty2 transcription in caffeine-treated and untreated yeast transformants. Deletion of the TEC1 gene had a slight effect on Ty2 transcription in untreated cells. But Ty2 transcription decreased more than twofold in $\Delta s g c 1$ mutant cells, indicating that this transcription activator is an essential component of Ty2 transcription. Caffeine treatment of $\Delta s g c 1$ and $\Delta t e c 1 \mathrm{mu}-$ tants also resulted in a two to threefold decrease in Ty2 transcription. However, when compared to the Ty2 expression level in the untreated wild type cells, Ty2 transcription is sixfold lower (185 units versus 29 units) in $\Delta$ sgc 1 mutants than the normal expression level in the wild type yeast (Table 3). Altogether, these results indicated that protein kinase $\mathrm{Snf} 1$ and transcription factor Sgc1 are required for the optimal level transcription of Ty2 under nutrient-replete standard growth conditions. Caffeine treatment of yeast cells represses Ty 2 expression approximately three to fourfold. Caffeine is a specific inhibitor of TOR1 kinase. A $p$-value which is less than $0.05((p<0.05)$ indicates that there are statistically significant differences in the expression levels of Ty2-917 gene fusions in the untreated and caffeine-treated yeast cells. However, it is clear that caffeine-dependent repression of Ty2 is largely independent of TORC1, Snf1 kinases, and transcription factors Sgc1 and Tec1.

\section{Effects of Autophagy Signaling on Enhancer Element of Ty2 retrotransposon}

Transcriptional regulatory regions of Ty2-917 are located both upstream and downstream of the transcription initiation site. The transcription activation region, an enhancer element, locates between 249 bp and 555bp of Ty2 (Figure 1). Downstream of an enhancer element, the negative regulatory region of Ty2 locates between 555 and 754 bp with respect to the first nucleotide of Ty2-917. An enhancer element activates transcription of any heterologous promoter when inserted upstream of the TATA box $(13,17)$. We wanted to analyze whether or not autophagy signaling acts on the enhancer element, independent from the negative regulatory region of Ty2. Transcription from the Ty2-555-LacZ reporter gene yielded 2158 units of activity as expected. The lack of negative regulatory region of Ty2 resulted in a more than tenfold increase in Ty2 transcription in normal growth conditions (Table 4). But caffeine treatment of the yeast

Table 4. Autophagy signaling targets Ty2 enhancer dependent transcription in wild type S. cerevisiae strain.

\begin{tabular}{lcc}
\hline Gene Fusions & $\begin{array}{c}\text { Growth Conditions and } \\
\boldsymbol{\beta} \text {-Galactosidase Activities * }( \pm \text { SD) }\end{array}$ & $\begin{array}{c}\text { Autophagy induction } \\
\text { (+8 } \mathbf{~ m M ~ C a f f e i n ) ~}\end{array}$ \\
\cline { 2 - 3 } & $\begin{array}{c}\text { Sc-Ura+\%2 Glucose } \\
\text { (Normal growth) }\end{array}$ & $965 \pm 77$ \\
\hline Ty2-555-LacZ** & $2158 \pm 200$ & $516 \pm 29$ \\
\hline p-ENC-LacZ & $852 \pm 55$ & \\
\hline $\begin{array}{l}\text { ( } \pm \text { SD): Standard deviations. } \\
\text { *Numbers indicate } \beta \text {-Galactosidase Units which are expressed in nano moles of ONPG cleaved per minute per mg of protein. }\end{array}$ & \\
\hline *Transcription levels were found significantly different $(p<0.05)$.
\end{tabular}


cells resulted in a nearly twofold decrease in the transcription of Ty2. Enhancer element-dependent transcription from a heterologous promoter yielded 852 units of activity in normally growing yeast transformants. Caffeine treatment of the yeast transformants resulted in a less than twofold decrease in enhancer element-dependent transcription. These results showed that autophagy activation by caffeine treatment targets regulatory factors, directly or indirectly, that are associated with transcription activation of Ty2, albeit not as high as in Ty2-754 that has a whole-length regulatory region (Table 4). A $p$-value that is less than $0.05(p<0.05)$ indicates that there are statistically significant differences in the expression levels of Ty2-555-LacZ gene fusions in the untreated and caffeine treated yeast cells.

\section{Amino Acid Starvation Represses Ty2-917 Transcription Independent of GCN4 Pathway}

Amino acid starvation that inactivates TOR1 signaling triggers the autophagy process in S. cerevisiae. We wanted to investigate if the Ty2 transcription will be affected by amino acid starvation. Previously constructed expression vectors that contain Ty2-555-LacZ and pEnh-LacZ gene fusions were transformed into F212 and F113 yeast strains. pST1 and pFN8X-n plasmids were also transformed into the same yeast strains as negative and positive controls, respectively. A portion of the yeast transformants was treated with 3-AT, a competitive inhibitor of the HIS3 gene product, to trigger amino acid starvation in these transformants.

Transcription of Ty2-555-LacZ decreased eightfold in 3-AT treated wild type S. cerevisiae F113 strain (1282 units to 162 units) (Table 5). Ty2 enhancer element-dependent transcription of a heterologous promoter ( $\mathrm{pEnh}$-LacZ) was repressed sixfold in amino acid starved yeast cells (Table 5). Amino acid starvation that inactivates TORC 1 was probably acting on the regulatory factors that bind to the Ty2 enhancer element since it also affects the enhancer activated transcription of a heterologous promoter. As expected, transcription of HIS4 gene activated threefold by amino acid starvation (from 812 units to 2388 units) (Table 5). Transcription from cloning vector PST1 that contains only the TATA box region is not affected by amino acid starvation.

It is well-known that transcription factor Gcn4 is activated by amino acid starvation in the wild type yeast strains (29). It was conceivable that Gcn4 could activate a kind of repressor protein that represses Ty 2 transcription in amino acid-starved yeast cells by acting on the Ty2 enhancer element either directly or indirectly. To test this possibility, we have measured the effect of amino acid starvation on the Ty2 transcription in gcn4 mutant S. cerevisiae F212 strain, isogenic to the wild type yeast strain. We found that Ty2 enhancer element-dependent transcription of a heterologous promoter and Ty2-555-LacZ was still repressible by amino acid starvation independent of the GCN4 pathway (Table 5). Transcription of Ty2-555-LacZ and the Ty2 enhancer-activated transcription of a heterologous promoter was repressed approximately four to fivefold in 3-AT treated gcn4 mutant F212 yeast strain (Table 5). As we expected, transcription of the His4-LacZ fusion gene in the pFN8x-n vector was activated in response to amino acid starvation in the wild type and decreased dramatically in the gcn4 mutant yeast cells due to the disruption of the GCN4 pathway. Transcription of the His4-LacZ fusion gene was further decreased in the amino acid-starved gcn4 mutant yeast cells (Table 5). Transcription of the UAS-less His4-LacZ fusion gene on PST1 is not affected by the gcn 4 mutation as expected. A $p$-value that is less than 0.05 $(p<0.05)$ indicates that there are statistically significant differences in the expression levels of Ty2-555-LacZ gene fusions in the untreated and 3-AT treated yeast cells. These results clearly indicated that amino acid starvation acts on Ty2 transcription, but is independent of the GCN4 pathway.

\section{GATA Factors Activity is Essential for Ty2 Transcription}

TOR1 kinase is the master regulator of autophagy in yeast. It also controls the activities of a group of transcription factors

Table 5. Amino acid starvation represses the Ty2-917 transcription independent of GCN4 pathway.

\section{Growth Conditions and \\ $\beta$-Galactosidase Activities * \pm SD)}

Gene fusions

Normal growth

pST1**

\begin{tabular}{l} 
p-ENC-LacZ \\
Ty2-555-LacZ** \\
\hline
\end{tabular}

Ty2-555-LacZ**

$1.8 \pm 0.5$
$309 \pm 25$
$1282 \pm 59$
$812 \pm 22$

\section{$( \pm \mathrm{SD})$ : Standard deviations.}

${ }^{*}$ Numbers indicate $\beta$-Galactosidase Units which are expressed in nano moles of ONPG cleaved per minute per mg of protein.

**Except pST1 expression levels, transcription levels were found significantly different $(p<0.05)$.

Amino acid starvation (+3-AT)

$1.9 \pm 0.7$

$162 \pm 12$

$2388 \pm 118$

ration

Normal growth

\section{F212 ( $\operatorname{gcn} 4)$}

Amino acid starvation (+3-AT) 
known as GATA factors (30). TOR1 kinase phosphorylates Gln3 and keeps it in the cytoplasm as Gln3-Ure2 complex in nutrient-replete conditions. Under nutrient starvation conditions, TOR kinase is inactivated and Gln3 is dephosphorylated. After that $\mathrm{Gln} 3$ is transferred to the nucleus and regulates nitrogen-regulated genes (30). GATA factors bind to the GATAA related sequences on the promoter regions of targeted genes. Gln3 and Gat1 are the activator factors. Dal80 and Gzf3 (Deh1) act as a transcriptional repressor on targeted genes depending on the nitrogen sources $(30,47)$.

In order to analyze whether the transcriptional activators GIn3 and Gat 1 have a function in Ty2 transcription, we have analyzed the transcription of Ty2-754-LacZ in the wild type and mutant yeast strain that do not contain Gln3 and Gat1 factors. Transcription of Ty2-754-LacZ gene fusion in the wild type yeast grown in a medium containing preferred nitrogen sources $(0.2 \%$ glutamine $)$ yielded 188 units of activity. When the yeast transformants are grown in a proline medium that results in the activation of $\mathrm{Gln} 3 / \mathrm{Gat} 1$, it results in the high level of activation in Ty2-754 gene expression. But, the expression level of Ty2-754-LacZ in the double mutant strain that does not contain the GIn3/Gat1 factor resulted in only 15 units of activity (Table 6). A significant level of decrease (from 309 units to 15 units) in the Ty2 transcription in the $\Delta g \ln 3 / \Delta$ gat 1 mutant strain clearly indicates that Ty2 transcription is controlled by GATA factors. A $p$-value that is less than $0.05(p<0.05)$ indicates that there are statistically significant differences in the expression levels of Ty2-754-LacZ gene fusions in the glutamine and proline treated yeast cells.

Ty2 and GCR1 Gene Promoter Regions have Multiple Putative Binding Sites for GATA Factors and Gcr1

It was previously shown that the GCR1 gene's transcription is autoregulated by itself. Gcr1, as a transcriptional activator, controls the regulation of $75 \%$ of S. cerevisiae genes (19). Transcription of Ty2 is also regulated by a Gcr1-dependent manner. Gcr1 binding sites (5'-CTTCC- 3 ') on the promoter regions of Ty2 and GCR1 gene were analyzed in detail in previous studies $(17,48)$.

After seeing the effects of the $\Delta g \ln 3 / \Delta g a t 1$ mutations on the transcription of Ty2, we have decided to analyze potential binding sites for the GATA factors on the Ty2 promoter regions. To analyze if there is any putative Gcr1 and GATA factor binding sites on the Ty2 and the GCR1 gene promoter regions, in-silico analyses were carried out using search tools and information provided by SGD and YEASTRACT databases. Analyses of the Ty2 promoter regions for the GATA factor binding sites revealed that it contains at least 6 putative binding sites (GATAAC) (Table 7). Interestingly, three of the GATA factor binding sites locate on the Crick strand (reverse direction to transcription) of Ty2 promoter regions, and two of the perfect consensus binding sites (GATAAT) for GATA factors locate within the negative regulatory region of Ty2 (Table 7). Additional binding sites for Gcr1 (5'-CTTCC- $\left.3^{\prime}\right)$ were also identified on the Ty2 promoter regions, in addition to previously determined Gcr1 binding sites (17). This result suggests that GATA factors (Gln3, Gat1, Deh80, and Gzf3) may directly bind to the Ty2 promoter.

Transcription of the GCR1 gene is highly dependent on the growth rate and growth conditions $(19,49)$. GCR1 mRNA completely diminished at a post-diauxic stage of yeast growth (19). Hence, there is a possibility that TORC1 may regulate GCR1 transcription, and transcription activator Gln3 (or other GATA factors) may involve in the regulation of GCR1 gene expression in a growth rate, and TORC1-dependent manner. Therefore, we have analyzed the promoter region of the GCR1 gene for the putative binding sites for GATA factors. In-silico analysis using YEASTRACT tools indicated that GCR1 gene promoter regions have at least 5 putative binding sites for GATA factors (Table 7). These results suggest that GATA factors are involved in the GCR1 gene regulation.

\section{DISCUSSION}

Gene expression in yeast Ty elements is controlled at the transcriptional and translational levels. Results presented in this study showed that transcription of the yeast retrotransposon Ty2-917 is repressed up to eightfold in amino acid starved yeast cells. Moreover, caffeine treatment of yeast cells also leads to three to fourfold repression of Ty2 transcription. However, our results indicated that caffeine treatment represses Ty2 transcription, independent from TORC1 and Snf1 pathways. Caffeine has multiple effects on eukaryotic cells. Although it is a specific inhibitor of TOR1 kinase, it also triggers ERAD mediated apoptosis (31). It also has a dramatic effect on DNA repair, cell wall integrity, and telomerase activity (31). Therefore, we think that the repression of Ty2 transcription resulting from caffeine

Table 6. GATA factors are essential for Ty2 transcription.

\section{$\beta$-Galactosidase Activities * $( \pm$ SD)}

\section{Nitrogen source}

\section{Wild type (YST182)}

$0.2 \%$ Glutamin $^{* *}$

$188 \pm 10$

$309 \pm 6$

\section{$\Delta$ gln3/gat1 mutant (YST184)}

$15 \pm 3$

$15 \pm 2$

$( \pm \mathrm{SD})$ : Standard deviations.

*Numbers indicate $\beta$-Galactosidase Units which are expressed in nano moles of ONPG cleaved per minute per mg of protein.

**Transcription levels were found significantly different $(p<0.05)$. 
Table 7. In-silico analysis of putative binding sites of Gcr1 and GATA factors on the Ty2 and GCR1 gene promoters.

\begin{tabular}{|c|c|c|c|}
\hline \multicolumn{2}{|c|}{$\begin{array}{l}\text { GATA factors' putative binding sites on the Ty2 promoter } \\
\text { region* }\end{array}$} & \multicolumn{2}{|c|}{$\begin{array}{l}\text { GATA factors' putative binding sites on the GCR1 promoter } \\
\text { regions* }\end{array}$} \\
\hline TF consensus binding sites** & Localization* & TF consensus binding sites** & Localization* \\
\hline GATAAT & $56-60(R)$ & GATTAG & $59-64(F)$ \\
\hline GATAAT & $136-142(F)$ & GATAAT & $163-167(R)$ \\
\hline GATAAT & $178-183(F)$ & GATAAT & $507-512(F)$ \\
\hline GATTAG & $425-430(F)$ & GATAAG & $610-615(R)$ \\
\hline GATAAT & $632-637(\mathrm{R})$ & GATAAA & $764-769(\mathrm{R})$ \\
\hline GATAAT & $654-658(R)$ & & \\
\hline \multicolumn{2}{|c|}{ Gcr1 binding sites on the Ty2 promoter region *** } & \multicolumn{2}{|c|}{ Gcr 1 binding sites on the GCR1 promoter regions *** } \\
\hline CTTCC & $115-119(\mathrm{R})$ & CTTCC & $112-116(F)$ \\
\hline ATTCC & 293-297 (R) & СTTCC & $472-476(F)$ \\
\hline СТTCC & $363-367(R)$ & СTTCC & $751-755(F)$ \\
\hline СTTCC & 401-405 (F) & СТTCC & 809-813 (F) \\
\hline СТTCC & 707-711 (F) & СТTCC & 893-897 (F) \\
\hline \multicolumn{4}{|c|}{$\begin{array}{l}\text { *Numbers for the GCR1 promoter region indicate the position of the first nucleotide of the consensus binding sequence of a transcription factor with respect to the } \\
\text { first nucleotide of the promoter region of GCR1 gene. Numbers for the binding sites on Ty2 indicate the position of the consensus binding sequences of relevant } \\
\text { transcription factors with respect to the first nucleotide of Ty2-917. (R) indicates the localization of given binding sites at reverse direction (Crick strand, template } \\
\text { strand), and (F) indicates the forward localization of relevant transcription factor consensus binding sites on the given promoters. } \\
\text { ** Consensus binding sequences of GATA factors and Gcr1 are taken from YEASTRACT database (www.yeastract.com). } \\
\text { ***Gcr1 binding sites on the Ty2 and GCR1 promoter regions were experimentally proven previously }(17,48) \text {. }\end{array}$} \\
\hline
\end{tabular}

treatment is the result of the combinatorial effects of caffeine on the various cellular components. We have already shown that activation of apoptosis by acetic acid treatment represses Ty1 and Ty2 transcription (21).

TOR1 kinase inhibits the autophagy process by directly acting on Atg13 factors. Nitrogen limitation and amino acid starvation inhibit TOR1 kinase activity. Therefore, it seems that nitrogen limitation and amino acid starvation are the better way of autophagy activation. Amino acid starvation leads to an eightfold decrease in Ty2 transcription. Amino acid starvation activates the expression of transcription activator Gcn4. Gcn4 is the master regulator of the general amino acid control (GAAC) pathway (29). However, our results clearly show that amino acid starvation has a negative effect on Ty2 transcription, independent of Gcn4. It is known that prolonged amino acid starvation causes a significant decrease in growth rate, and leads to cell cycle arrest in the G1 stage (50). Previously we have shown that transcription of Ty2 is also regulated in a growth rate-dependent manner. Its transcription decreases by more than $50 \%$ in the stationary stage (51). It was also shown that Gcr1 itself controls cell growth by acting on nutrient-regulated gene expression on a global level (49). Gcr1 transcription is autoregulated by itself and its transcription completely stops once the glucose is consumed in the growth medium at the late logarithmic/diauxic stage (19). Hence it is also possible that prolonged incubation of the yeast cells during amino acid starvation, which results in cell cycle arrest, and consumption of glucose in the growth medium leads to inhibition of GCR1 expression, and this results in the downregulation of Ty2 transcription in the yeast cells.

Gcr1 is a phosphoprotein and recent evidence indicated that Gcr1 isoforms are phosphorylated and pyrophosphorylated depending on the growth conditions $(19,49,52)$. It was shown that once Gcr1 is pyrophosphorylated by inositol hexakisphosphate kinase (KCSI), its transcription decreases at a significant level (52). TOR1 kinase and inositol hexakisphosphates synergistically control nutrient-sensing and growth control. Moreover, new evidence indicates that increased levels of 5-diphosphoinositol pentakisphosphate upon stress conditions result in the increased nuclear localization of the transcriptional activators Msn2 and Gln3 (53). New reports indicate that inositol hexakisphosphates also promote autophagy in tissue culture cells (54). Hence, repression of Ty2 transcription in amino acid-starved yeast cells may result from the combinatorial effects of the autophagy signaling and nutrient-dependent down regulation of Gcr1 activity.

Nitrogen limitation, or use of poor nitrogen sources such as urea, proline, or allantoin, also inhibit TOR1 kinase activity (30, 47). TORC1 phosphorylates Gln3 in nutrient-replete conditions. 
Phosphorylated Gln3 forms a heterodimer with Ure2 and remains in the cytoplasm. When TOR1 kinase activity is inhibited, Gln3 dephosphorylates and enters nuclei to activate targeted genes $(30,47)$. But recent evidence indicated that $G \ln 3$ also functions independent of TOR kinase activity, independent from Ure2 interaction. Therefore, there is always GIn3-dependent activation of targeted yeast genes, albeit at low levels. Growth of yeast cells in proline medium as a sole nitrogen source leads to approximately threefold activation of Ty2 transcription. Lack of same level activity in $\Delta g / n 3 / \Delta g a t 1$ mutants reveals that even under standard growth conditions (in glutamine medium), GATA factors GIn3/Gat1 are the significant parts of Ty 2 transcription activation components. This result also emphasizes that in addition to transcription factor Gcr1, Ty2 transcription is dependent on Gln3/Gat1.

Genetic evidence that we have provided using $\Delta g \ln 3 / \Delta$ gat mutant strains indicates that at least two of the GATA factors, GIn3 and Gat1, are involved in the activation of Ty2 transcription. Dal80 is another member of the GATA group transcription factors. Contrary to Gln3/Gat1, Dal80 is a repressor protein. Recently published results indicate that Dal80 represses a large group of genes in yeast genes. It turned out that two of these genes are TEC1 and SGC1 (TYE7) (55). This new evidence suggests that Dal80 could also repress Ty2 transcription indirectly by interfering with transcription of the TEC1 and SGC1 genes. GATA factors directly bind to GATAA related sequences on the targeted promoters. Gln3 and Gat 1 are activator factors while Dal80 and Gzf3 (Deh1) are the repressor group of GATA family transcription factors. It was proposed that the GATA factor competes for their binding sites on the targeted promoters (56).

a-) Nutrient replete growth conditions TORC1 active

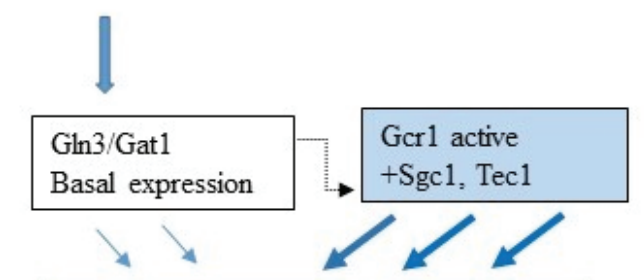

High level transcription of Ty2

b-) Amino acid starvation (slow growth, G0 stage)

TORC 1 inactive

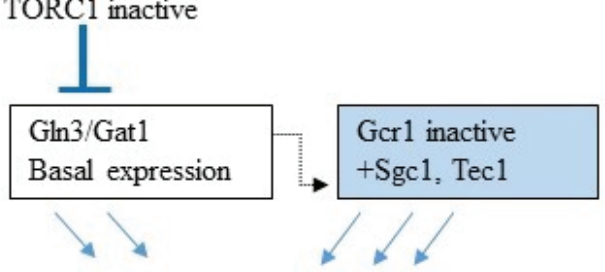

Low level transcription of Ty2
In-silico analysis of the Ty2 regulatory region for the transcription factor binding sites using the YEASTRACT database confirms that there are multiple potential binding sites for GATA factors in the regulatory region of Ty2. It is also possible that Dal80 or Gzf3 is one of the regulatory factors involving Ty2 repression by interacting with GATAA sequences in the negative regulatory region of Ty2. Our in-silico analyses results indicated that transcription of the GCR1 gene might be regulated by GATA factors. Knowing that the GCR1 gene transcription is regulated growth rate and growth stage dependent-manner, it seems reasonable to suggest that TOR1 kinase affects the expression of Gcr1-dependent gene regulation by modulating the GCR1 gene transcription via GATA factors. Nonetheless, functional analysis of GATA factors' involvement in the regulation of GCR1 transcription is necessary to experimentally validate these hypotheses. Deletion of GATA factor binding sites on the GCR1 promoter, in-vitro binding assays (such as EMSA and/or DNasel Footprint) with the DNA fragments from the GCR1 promoter region and purified GATA factors would provide conclusive in-vivo and intro evidence as to whether GATA factors control the transcription of GCR1 gene and Ty2 retrotransposon $(17,37,56)$.

Based on our experimental and in-silico analyses results, we propose the following model on the regulation of Ty2 transcription in response to the autophagy signaling. Under nutrient-replete conditions at the logarithmic stage of the cell growth, in which TORC 1 complex is active and autophagy is repressed, Ty2 transcription is activated mainly by Gcr1, Sgc1, and Tec1. Gln3, Gat1 which are expressed at a basal level, might also contribute to Ty2 transcription in nutrient-replete growth conditions (Figure 2a). In amino acid starved yeast cells, the growth rate slows and

c- ) Nitrogen starvation (proline)(glucose replent) TORC1 inactive
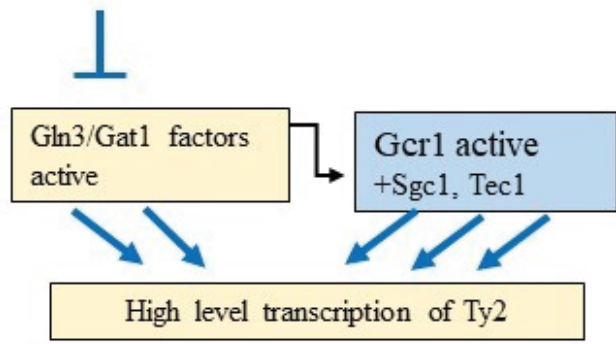

d-) $\Delta \operatorname{gln} 3 / \Delta$ gat1 deletion mutations (slow growth) (Glucose replete, glutamine or proline)

\begin{tabular}{|l|}
\hline Gln $3 /$ Gat 1 factors \\
Not expressed
\end{tabular}

Gcr1 low level expression + Sgc1, Tec1

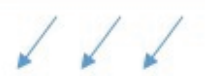

Figure 2. Proposed model on the regulation of Ty2 transcription in response to various growth conditions in yeast. 
yeast cells enter G0 stage as the result of prolonged starvation. This situation results in the complete inactivation of the GCR1 expression. TOR1 kinase is inactivated and the autophagy process initiates due to amino acid starvation. This metabolic situation leads to a significant level decrease in Ty2 transcription. On the other hand, caffeine treatment also inactivates TORC1. But, in caffeine-treated yeast cultures, the nitrogen source and glucose are not consumed completely. Therefore, we think that the autophagy process does not operate at full scale in the caffeine-treated, nutrient-replete yeast cells during the 5-hour incubation period. Under these circumstances, Ty2 transcription occurs at moderate to low levels using relevant transcription factors (Figure 2b). If the cells are grown in proline medium as sole nitrogen sources in glucose replete medium, TORC1 is inactivated. This results in the full-scale activation of GATA factors Gln3/Gat1. In turn, these GATA factors activate Ty2 transcription by directly targeting the Ty2 promoter, and also by further acti-

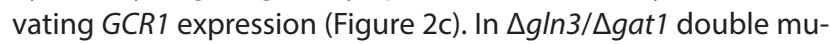
tant yeast, Ty 2 transcription decreases at a significant level. This decrease might be the result of multiple events, such as lack of functional active Gln3/Gat1 factors and low-level expression of GCR1 or slow growth phenotypes of the mutant strain $(49,57)$ (Figure 2d).

Overall, it appears that Ty2 retrotransposon acquired multiple regulatory factors to adjust its expression levels in response to various metabolic changes for its existence in its host cells. The involvement of the autophagy process in viral infections amplification and/or eradication underscores the different applications of autophagy in clinical fields $(34,35)$. It seems that regulation of the copy number of Ty retrotransposons by degradation of Ty-VLP within autophagosomes and repression of Ty2 transcription in response to autophagy signals makes Ty retrotransposons a potentially good model system to analyze the effects of the autophagy process on the viral propagation in eukaryotic cells.

\section{CONCLUSION}

Previously, it was shown that activation of autophagy condition results in the elimination of cytoplasmic Ty2-VLP by autophagosomes. Results of this study indicated that the transcription of retrotransposon Ty2 is also repressed in response to autophagy signaling. It seems that autophagy signaling represses Ty2 transcription by multiple mechanisms including direct binding of repressors and inhibition of activator functions such as Gcr1, Gln3, and Gat1 that are involved in Ty2 transcription. In addition to TY-VLP elimination by autophagy, nutrient limitations could also restrict Ty2 retrotransposon's transcription to limit Ty mobility in the yeast genome under stressful, nutrient-restricted growth conditions.

Informed Consent: Written consent was obtained from the participants.

Peer Review: Externally peer-reviewed.

Author Contributions: Conception/Design of Study- S.T., C.C.;
Data Acquisition- C.C., S.T., T.K.; Data Analysis/InterpretationS.T., C.C.; Drafting Manuscript- S.T., C.C.; Critical Revision of Manuscript- S.T.; Final Approval and Accountability- C.C., S.T., T.K.

Conflict of Interest: Authors declared no conflict of interest.

Financial Disclosure: Authors declared no financial support.

\section{REFERENCES}

1. Kim JM, Vanguri S, Boeke JD, Gabriel A, Voytas DF. Transposable Elements and Genome Organization: A Comprehensive survey of retrotransposons revealed by the complete Saccharomyces cerevisiae genome sequence. Genome Res 1998; 8: 464-78.

2. Boeke J, Garfinkel DJ, Styles CA, Fink G. Ty elements transpose through an RNA intermediate. Cell 1985; 40: 491-500.

3. Curcio MJ, Lutz S, Lesage P. The Ty1 LTR-retrotransposon of budding yeast, Saccharomyces cerevisiae. Microbiol Spectr 2015; 3: 1-35.

4. Capy P. Classification and nomenclature of retrotransposable elements. Cytogenet Genome Res 2005; 110: 457-61.

5. Roeder GS, Farabaugh PJ, Chalef DT, Fink GR. The origin of gene instability in yeast. Science 1980; 209: 1375-80.

6. Garfinkel DJ, Tucker JM, Saha A, Nishida Y, Pachulska-Wieczorek K, Błaszczyk $L$, et al. A self-encoded capsid derivative restricts Ty1 retrotransposition in Saccharomyces. Curr Genet 2016; 62: 321-9.

7. Saha A, Mitchell JA, Nishida Y, Hildreth JE, Ariberre JA, Gilbert WV, et al. A trans-dominant form of Gag restricts Ty1 retrotransposition and mediates copy number control. J Virol 2015; 89: 3922-38.

8. Curcio MJ, Hedge AM, Boeke JD, Garfinkel DJ. 1990. Ty RNA levels determine the spectrum of retrotransposition events that activate gene expression in Saccharomyces cerevisiae. Mol Gen Genet 1990; 220: 213-21.

9. Belcourt MF, Farabaugh PJ. Ribosomal frameshifting in the yeast retrotransposon Ty: tRNAs induce slippage on a 7 nucleotide minimal site. Cell 1990; 62: 339-52.

10. Farabaugh PJ. Programmed translational frameshifting. Microbiol Rev 1996; 60: 103-134.

11. Farabaugh PJ, XB. Liao, Belcourt M, Zhao H, Kapakos J, Clare J. Enhancer and silencer-like sites within the transcribed portion of a Ty2 transposable element of S. cerevisiae. Mol Cell Biol 1989; 9: 4824-34.

12. Farabaugh PJ, Vimaladithan A, Türkel $S$, Johnson $R$, Zhao $H$. Three downstream sites repress transcription of a Ty2 retrotransposon in Saccharomyces cerevisiae. Mol Cell Biol 1993; 13: 2081-90.

13. Türkel S, Farabaugh PJ. Interspersion of an unusual GCN4 activation site with a complex transcriptional repression site in Ty elements of Saccharomyces cerevisiae. Mol Cell Biol 1993; 13: 2091103.

14. Laloux I, Dubois E, Dewerchin M, Jacobs E. TEC1, a gene involved in the activation of Tyl and Tyl-mediated gene expression in Saccharomyces cerevisiae: Cloning and molecular analysis. Mol Cell Biol 1990; 10: 3541-50.

15. Löhning C, Ciriacy M. The TYE7 gene of Saccharomyces cerevisiae encodes a putative bHLH-LZ transcription factor required for Ty1-mediated gene expression. Yeast 1994; 10: 1329-39.

16. Türkel S, Yenice B. Analysis of the effects of chromatin modifying complexes on the transcription of retrotransposon Ty2-917 in Saccharomyces cerevisiae. Turk J Biol 2006; 30: 101-6.

17. Türkel S, Liao XB, Farabaugh PJ. Gcr1-dependent transcriptional activation of yeast retrotransposon Ty2-917. Yeast 1997; 13: 91730. 
18. Cha S, Hong CP, Kang HA, Hahn J-S. Differential activation mechanisms of two isoforms of Gcr1 transcription factor generated from spliced and un-spliced transcripts in Saccharomyces cerevisiae. Nucleic Acids Res 2021; 49: 745-59.

19. Hossain MA, Claggett JM, Edwards S, Shi A, Pennebaker S, Cheng $M$, et al. Post-transcriptional regulation of Gcr1 expression and activity are crucial for metabolic adjustment in response to glucose availability. Mol Cell 2016; 62: 346-58.

20. Suzuki K, Morimoto M, Kondo C, Ohsumi Y. Selective autophagy regulates insertional mutagenesis by the Ty1 retrotransposon in Saccharomyces cerevisiae. Developmental Cell 2011; 21: 358-65.

21. Çolakoğlu C, Türkel S. Apoptosis signaling pathway regulates the gene expression in the yeast retrotransposons Ty1 and Ty2. Eur J Biology 2020; 79: 36-42.

22. Ohsumi Y. Historical landmarks of autophagy research. Cell Research 2014; 24: 9-23.

23. Suzuki K, Ohsumi Y. Molecular machinery of autophagosome formation in yeast, Saccharomyces cerevisiae. 2007; FEBS Lett 581: 2156-61.

24. Yin Z, Pascual C, Klionsky DJ. Autophagy: machinery and regulation. Microb Cell 2016; 3: 588-96.

25. Delorme-Axford E, Daniel J. Klionsky DJ. Transcriptional and post-transcriptional regulation of autophagy in the yeast Saccharomyces cerevisiae. J Biol Chem 2018; 293: 5396-403.

26. Wen X, Klionsky DJ. An overview of macroautophagy in yeast. J Mol Biol 2016; 428: 1681-99.

27. Kamada Y, Funakoshi T, Shintani T, Nagano K, Ohsumi M, Ohsumi Y. Tor-mediated induction of autophagy via an Apg1 protein kinase complex. J Cell Biol 2000; 150: 1507-13.

28. Yoshiaki Kamada Y, Yoshino K-İ, Kondo C, Kawamata T, Oshiro N, Yonezawa K, et al. Tor directly controls the Atg1 kinase complex to regulate autophagy. Mol Cell Biol 2010; 30: 1049-58.

29. Hinnebusch, AG, Natarajan K. 2002. Gcn4p, a master regulator of gene expression, is controlled at multiple levels by diverse signals of starvation and stress. Eukaryotic Cell 2002; 1: 22-32.

30. Cooper TG. Transmitting the signal of excess nitrogen in Saccharomyces cerevisiae from the Tor proteins to the GATA factors: connecting the dots. FEMS Microbiol Rev 2002; 26: 223-38.

31. Ruta LL, Farcasanu IC. Saccharomyces cerevisiae and caffeine implications on the eukaryotic cell. Nutrients 2020; 12: 2440; doi:10.3390/nu12082440.

32. Galluzzi L, Baehrecke EH, Ballabio A, Boya P, Bravo-San Pedro JM, Cecconi $F$, et al. Molecular definitions of autophagy and related processes. EMBO J 2017; 13: 1811-36.

33. Scott SV, Guan J, Hutchins MU, Kim J, Klionsky DJ. Cvt19 is a receptor for the Cytoplasm-to-Vacuole targeting pathway. Mol Cell 2001; 7: 1131-41.

34. Choi Y, Bowman JW, Jung JU. Autophagy during viral infection- a double-edged sword. Nat Rev Microbiol 2018; 16: 341-54.

35. Campbell GR, Spector SA. Induction of autophagy to achieve a human immunodeficiency virus type 1 cure. Cells 2021; 10: 1798. doi. org/10.3390/cells10071798.

36. Brachmann CB, Davies A, Cost GJ, Caputo E, Li J, Hieter P, Boeke JD. Designer deletion strains derived from Saccharomyces cerevisiae S288C: a useful set of strains and plasmids for PCR-mediated gene disruption and other applications. Yeast 1998; 14: 115-32.

37. Scherens B, Feller A, Vierendeels F, Messenguy F, Dubois E. Identification of direct and indirect targets of the Gln 3 and Gat 1 activators by transcriptional profiling in response to nitrogen availability in the short and long term. FEMS Yeast Research 2006; 6: 777-91.

38. Nagawa F, Fink GR. 1985. The relationship between the "TATA" sequence and transcription initiation sites at the HIS4 gene of Saccharomyces cerevisiae. Proc Natl Acad Sci (USA) 1985; 82: 8557-61.
39. Arndt KT, Styles C, Fink GR. Multiple global regulators control HIS4 transcription in Yeast. Science 1987; 237: 874-80.

40. Christianson TW, Sikorski RS, Dante M, Shero JH, Hieter P. 1992. Multifunctional yeast high-copy-number shuttle vectors. Gene 1992; 110: 119-22.

41. Gietz R., Schiestl R. High-efficiency yeast transformation using the LiAc/SS carrier DNA/PEG method. Nat Protoc 2007; 2: 31-4.

42. Kuranda K, Leberre V, Sokol S, Palamarczyk G, Francois J. Investigating the caffeine effects in the yeast Saccharomyces cerevisiae brings new insights into the connection between TOR, PKC and Ras/cAMP signalling pathways. Molecular Microbiol 2006; 61: 1147-66.

43. Guarente L, Patashe M. Fusion of Escherichia coli lacZ to the cytochrome c gene of Saccharomyces cerevisiae. Proc Natl Acad Sci (USA) 1981; 78: 2199-203.

44. Lowry OH, Rosebrough NJ, Farr AL, Randall RJ. Protein measurement with the Folin phenol reagent. J Biol Chem 1951; 193: 265-75.

45. Schmelzle T, Hall MN. TOR, a central controller of cell growth. Cell 2000; 103: 253-262.

46. Coccetti P, Nicastro R, Tripodi F. Conventional and emerging roles of the energy sensor Snf1/AMPK in Saccharomyces cerevisiae. Microb Cell 2018; 5: 482-94.

47. Georis I, A. Feller A, Tate JJ, Cooper TG, Dubois E. Nitrogen catabolite repression-sensitive transcription as a readout of Tor pathway regulation: the genetic background, reporter gene and GATA factor assayed determine the outcomes. Genetics 2009; 181: 861-74.

48. Sasaki H, Kishimoto T, Mizuno T, Shinzato T, Uemura H. Expression of GCR1, the transcriptional activator of glycolytic enzyme genes in the yeast Saccharomyces cerevisiae, is positively autoregulated by Gcr1p. Yeast 2005; 22: 305-19.

49. Barbara KE, Haley TM, Willis KA, Santangelo GM. The transcription factor Gcr1 stimulates cell growth by participating in nutrient-responsive gene expression on a global level. Mol Genet Genomics 2007; 277: 171-88.

50. Gonzalez A, Hall MN. Nutrient sensing and TOR signaling in yeast and mammals. EMBO J 2017; 36: 397-408.

51. Türkel S, Bayram Ö, Arık E. Glucose signaling pathway and growth conditions regulate gene expression in retrotransposon Ty2. $Z$. Naturforsch 2009; 64 c: 526-32.

52. Szijgyarto Z, Garedew A, Azevedo C, Saiardi A. Influence of inositol pyrophosphates on cellular energy dynamics. Science 2011; 334: 802-5.

53. Morrissette VA, Rolfes RJ. The intersection between stress responses and inositol pyrophosphates in Saccharomyces cerevisiae. Curr Genet 2020; 66: 901-10.

54. Nagata E, Saiardi A, Tsukamoto H, Satoh T, Itoh Y, Itoh J, et al. Inositol hexakisphosphate kinases promote autophagy. Int J Biochem Cell Biol 2010; 42: 2065-71.

55. Ronsmans A, Wery M, Szachnowski U, Gautier C, Descrimes M, Dubois $E$, et al. Transcription-dependent spreading of the Dal80 yeast GATA factor across the body of highly expressed genes. PLoS Genet 2019; 15: e1007999. doi.org/10.1371/journal.pgen.1007999.

56. Coffman JA, Rai R, Loprete DM, Cunningham T, Svetlov V, Cooper TG. Cross regulation of four GATA factors that control nitrogen catabolic gene expression in Saccharomyces cerevisiae. J Bacteriol 1997; 179: 3416-29.

57. Lopez MC, Baker HV. Understanding the growth phenotype of the yeast $\mathrm{gcr} 1$ mutant in terms of global genomic expression patterns. J Bacteriol 2000; 182: 4970-8. 